\title{
Optimal Control of Cervical Cancer Model with Vaccination and Screening
}

\author{
Karunia Theda Kristanti ${ }^{*}$, Trisilowati $^{2}$, Agus Widodo \\ ${ }^{1}$ Master Program of Mathematics, Faculty of Mathematics and Natural Sciences, University of Brawijaya, Malang, \\ Indonesia \\ 2Department of Mathematics, Faculty of Mathematics and Natural Sciences, University of Brawijaya, Malang, Indonesia
}

\begin{abstract}
In this paper, an optimal control problem of a cervical cancer model with vaccination and screening as controls is discussed. This vaccine can stimulate the immune system to produce antibodies that can prevent the occurrence of human papillomavirus (HPV) infections, while screening is used as secondary prevention of early detection of cervical cancer cells so that treatment can begin immediately. The models were divided into two compartments, females and males. The female's compartment consists of susceptible, vaccinated, infected, screening, cervical cancer, and recovered females. Meanwhile, the male's compartment consists of susceptible, infected, and recovered males. The purpose of this optimal control was to minimize the number of infected females, infected males, and cervical cancer, as well as to minimize the cost of the controls. Optimal control was obtained by using the Pontryagin principle. Furthermore, an optimal control problem was solved numerically using the Forward-Backward Sweep method to determine the effect of vaccination and screening on the model. The results indicate that vaccination and screening as controls are effective in reducing the subpopulation of HPV infection, which can further reduce the occurrence of cervical cancer.
\end{abstract}

Keywords: cervical cancer, vaccination, screening, optimal control

\section{INTRODUCTION}

Cervical cancer is abnormal growth and development of cervix cells and causes some abnormal organ function [1]. Cervical cancer generally attacks the sexually active female. The cause of cervical cancer is Human Papillomavirus (HPV) infection, especially types 16 and 18 . This virus can be transmitted through sexual activity changing partners [2]. The occurrence of cervical cancer is also increased in the population with several factors, including late diagnosis, low socioeconomic status, limited resources, limited infrastructure, and low degrees of education [3].

Cervical cancer can be prevented by HPV vaccinations and screening examinations, such as the Pap smear test or IVA [4]. Vaccination is given to stimulate the immune system to produce antibodies. Further, these antibodies will prevent HPV from infecting cervical cells. Three types of vaccines can be used, namely bivalent, quadrivalent, and nonavalent. Meanwhile, the Pap smear test and the IVA test are also crucial in suppressing the incidence of cervical cancer immediately. This test is an early attempt to find

\footnotetext{
${ }^{*}$ Correspondence address:

Karunia Theda Kristanti

Email : kristantikarunia@gmail.com

Address : Dept. Mathematics, University of Brawijaya, Veteran Malang, Malang 65145
}

out the presence of cervical cancer cells so that treatment can be done.

Related to the transmission of HPV infection and cervical cancer, in 2014, Pongsumpun [5] modeled the spread of cervical cancer in the female population. This study found that every woman could be infected by HPV when the occurrence of HPV infection is high. Therefore, a woman's risk of cervical cancer will also be high. In contrast to the model discussed by Pongsumpun, Malik et al. in 2016 [6] examined the optimal control of the spread of HPV in the presence of vaccination. The model consists of the female's compartment and the male's compartment. It is assumed that only the population of the susceptible female who gets vaccinated. There are three types of vaccines used as controls, namely bivalent, quadrivalent, and nonavalent vaccines.

Furthermore, Sado [7] discussed the model of the spread of cervical cancer in the female population by adding the parameters of vaccination. Based on these results, the vaccine can reduce and control the transmission of cervical cancer due to HPV infection. Regarding the model involving vaccination and screening, Saldana et al. [8] discussed the optimal control of HPV infection transmission models without involving cervical cancer. The results of the study of Saldana et al. explain that vaccination and 
screening can reduce the level of infected individuals in both the female and male subpopulations.

This research constructs a mathematical model of cervical cancer by vaccination and screening that modify the model of Malik et al. and Saldana et al. It was assumed that every susceptible female can have only one type of vaccine. In this model, cervical cancer can occur due to sexual interactions between female and male. Next, we investigated the effect of this interaction on both the female and male populations. Therefore, the population was divided into female and male compartments.

\section{MATERIAL AND METHOD}

The model of cervical cancer was constructed by involving control vaccination $\left(\boldsymbol{u}_{1}\right)$ and screening $\left(\boldsymbol{u}_{2}\right)$. Next, an optimal control problem was carried out with the following steps.

a. Construct a cervical cancer model with vaccination and screening as controls.

b. Define the objective functional to minimize the number of populations infected with HPV infection.

c. Apply the Pontryagin principle to solve the optimal control problem.

d. Determine the state, adjoint, and stationary conditions.

e. Perform numerical simulations using the Sweep Forward and Backward method using MATLAB software.

\section{RESULT AND DISCUSSION}

\section{Construction Model}

In the cervical cancer model, the population was divided into the female population and the male population. The female population was divided into six subpopulation: susceptible female $\left(S_{f}\right)$ consists of individuals who have not vaccinated, vaccinated females $(V)$ consists of individuals who carry out vaccinations, infected females $\left(I_{h}\right)$ consists of individuals infected with HPV, screening female $\left(I_{s c}\right)$ consists of individuals who carry out prevention with Pap smear or IVA test, cervical cancer females $(C)$ consists of individuals with cervical cancer, recovered females $\left(R_{f}\right)$ consists of individuals who recover from the infection and having cleared from infection due to treatment or leaving the sexual activity.

Meanwhile, the male population is divided into three subpopulations: susceptible male $\left(S_{m}\right)$ consists of individuals who have a chance of being infected with HPV, infected male $(J)$ consists of individual infected with HPV, and recovered male $\left(R_{m}\right)$ consists of individuals recovering from infection and having cleared from infection. The model is constructed under the assumptions,

1. Only a subpopulation of the susceptible female is given vaccination using one type of vaccine, such as bivalent, quadrivalent, or nonavalent vaccine.

2. Screening is also only done by a subpopulation of infected females.

3. The transmission of HPV infection is caused by an interaction between susceptible subpopulation and infected subpopulation.

4. Individual of recovered subpopulation who has been cleared of HPV infection will be able to lose the immunity and move to a susceptible population.

The diagram of the model can be described as in Figure 1. Based on the assumption above, the model can be written in the form of differential equations:

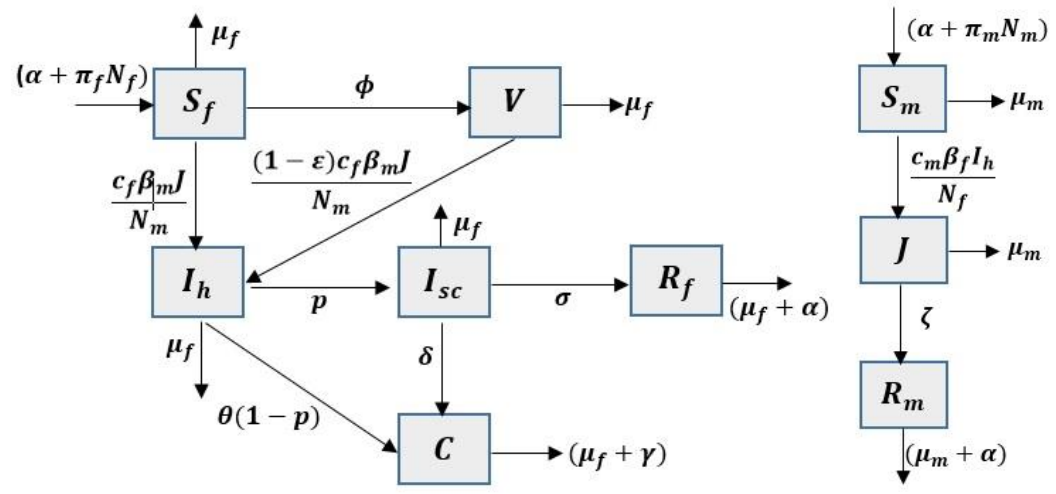

Figure 1. Flow diagram of the model 


$$
\begin{array}{rlrl}
\frac{d S_{f}(t)}{d t}= & \pi_{f} N_{f}(t)-\phi S_{f}(t)-\frac{c_{f} \beta_{m} J(t) S_{f}(t)}{N_{m}(t)} & & +\alpha \bar{R}_{m}(t), \\
& -\mu_{f} S_{f}(t)+\alpha R_{f}(t), & \frac{d \bar{J}(t)}{d t}=c_{m} \beta_{f} \bar{I}_{h}(t) \bar{S}_{m}(t)-\zeta \bar{J}(t)-\pi_{m} \bar{J}(t), \\
\frac{d V(t)}{d t}= & \phi S_{f}(t)-\mu_{f} V(t)-(1-\varepsilon) \frac{c_{f} \beta_{m} J(t) V(t)}{N_{m}}, & \frac{d \bar{R}_{m}(t)}{d t}=\zeta \bar{J}(t)-\pi_{m} \bar{R}_{m}(t)-\alpha \bar{R}_{m}(t) .
\end{array}
$$$$
\frac{d I_{h}(t)}{d t}=\frac{c_{f} \beta_{m} J(t) S_{f}(t)}{N_{m}(t)}+(1-\varepsilon) \frac{c_{f} \beta_{m} J(t) V(t)}{N_{m}(t)}
$$$$
-p I_{h}(t)-\mu_{f} I_{h}(t)-\theta(1-p) I_{h}(t),
$$$$
\frac{d I_{s c}(t)}{d t}=p I_{h}(t)-\sigma I_{s c}(t)-\mu_{f} I_{s c}(t)-\delta I_{s c}(t),
$$$$
\frac{d C(t)}{d t}=\delta I_{s c}(t)+\theta(1-p) I_{h}(t)-\left(\mu_{f}+\gamma\right) C(t),
$$$$
\frac{d R_{f}(t)}{d t}=\sigma I_{s c}(t)-\mu_{f} R_{f}(t)-\alpha R_{f}(t),
$$$$
\frac{d S_{m}(t)}{d t}=\pi_{m} N_{m}(t)-\frac{c_{m} \beta_{f} I_{h}(t) S_{m}(t)}{N_{f}(t)}-\mu_{m} S_{m}(t)
$$$$
+\alpha R_{m}(t) \text {, }
$$$$
\frac{d J(t)}{d t}=\frac{c_{m} \beta_{f} I_{h}(t) S_{m}(t)}{N_{f}(t)}-\zeta J(t)-\mu_{m} J(t),
$$$$
\frac{d R_{m}(t)}{d t}=\zeta J(t)-\mu_{m} R_{m}(t)-\alpha R_{m}(t),
$$$$
\text { where }
$$$$
N_{f}(t)=S_{f}(t)+V(t)+I_{h}(t)+I_{s c}(t)+C(t)+R_{f}(t) \text {, }
$$$$
\text { and }
$$$$
N_{m}(t)=S_{m}(t)+J(t)+R_{m}(t) \text {. }
$$

The description of the parameters used in the model is described in Table 1. We normalize the equations (1) in terms of the proportions

$$
\begin{aligned}
& \bar{S}_{f}(t)=\frac{S_{f}(t)}{N_{f}(t)}, \bar{V}(t)=\frac{V(t)}{N_{f}(t)}, \bar{I}_{h}(t)=\frac{I_{h}(t)}{N_{f}(t)^{\prime}} \\
& \bar{I}_{s c}(t)=\frac{I_{s c}(t)}{N_{f}(t)}, \bar{C}(t)=\frac{C(t)}{N_{f}(t)}, \bar{R}_{f}(t)=\frac{R_{f}(t)}{N_{f}(t)^{\prime}}, \\
& \bar{S}_{m}(t)=\frac{S_{m}(t)}{N_{m}(t)}, \bar{J}(t)=\frac{J(t)}{N_{m}(t)}, \bar{R}_{m}(t)=\frac{R_{m}(t)}{N_{m}(t)} .
\end{aligned}
$$

Then normalized system becomes,

$$
\begin{aligned}
\frac{d \bar{S}_{f}(t)}{d t}= & \pi_{f}-\phi \bar{S}_{f}(t)-c_{f} \beta_{m} \bar{J}(t) \bar{S}_{f}(t) \\
& -\pi_{f} \bar{S}_{f}(t)+\alpha \bar{R}_{f}(t) \\
\frac{d \bar{V}(t)}{d t}= & \phi \bar{S}_{f}(t)-(1-\varepsilon) c_{f} \beta_{m} \bar{J}(t) \bar{V}(t)-\pi_{f} \bar{V}(t), \\
\frac{d \bar{I}_{h}(t)}{d t}= & c_{f} \beta_{m} \bar{J}(t) \bar{S}_{f}(t)+(1-\varepsilon) c_{f} \beta_{m} \bar{J}(t) \bar{V}(t) \\
& -p \bar{I}_{h}(t)-\theta(1-p) \bar{I}_{h}(t)-\pi_{f} \bar{I}_{h}(t) \\
\frac{d \bar{I}_{s c}(t)}{d t}= & p \bar{I}_{h}-\sigma \bar{I}_{s c}(t)-\delta \bar{I}_{s c}(t)-\pi_{f} \bar{I}_{s c}(t) \\
\frac{d \bar{C}(t)}{d t}= & \delta \bar{I}_{s c}(t)+\theta(1-p) \bar{I}_{h}(t)-\gamma \bar{C}(t) \\
& -\pi_{f} \bar{C}(t) \\
\frac{d \bar{R}_{f}(t)}{d t}= & \sigma \bar{I}_{s c}(t)-\pi_{f} \bar{R}_{f}(t)-\alpha \bar{R}_{f}(t) \\
\frac{d \bar{S}_{m}(t)}{d t}= & \pi_{m}-c_{m} \beta_{f} \bar{I}_{h}(t) \bar{S}_{m}(t)-\pi_{m} \bar{S}_{m}(t)
\end{aligned}
$$

Providing control in the form of vaccination as primary prevention is carried out in susceptible populations to prevent the occurrence of HPV infection. Meanwhile, secondary prevention in the form of screening was done as an early effort to find out the presence of abnormal cells that can cause cervical cancer. The formulation model of cervical cancer transmission with controls in the form of vaccination $\left(u_{1}\right)$ and screening $\left(u_{2}\right)$ is presented as follows.

$$
\begin{aligned}
& \frac{d \bar{S}_{f}(t)}{d t}=\pi_{f}-u_{1}(t) \bar{S}_{f}(t)-c_{f} \beta_{m} \bar{J}(t) \bar{S}_{f}(t) \\
& -\pi_{f} \bar{S}_{f}(t)+\alpha \bar{R}_{f}(t), \\
& \frac{d \bar{V}(t)}{d t}=u_{1}(t) \bar{S}_{f}(t)-(1-\varepsilon) c_{f} \beta_{m} \bar{J}(t) \bar{V}(t) \\
& -\pi_{f} \bar{V}(t), \\
& \frac{d \bar{I}_{h}(t)}{d t}=c_{f} \beta_{m} \bar{J}(t) \bar{S}_{f}(t)+(1-\varepsilon) c_{f} \beta_{m} \bar{J}(t) \bar{V}(t) \\
& -u_{2}(t) \bar{I}_{h}(t)-\theta\left(1-u_{2}(t)\right) \bar{I}_{h}(t)-\pi_{f} \bar{I}_{h}(t), \\
& \frac{d \bar{I}_{s c}(t)}{d t}=u_{2}(t) \bar{I}_{h}-\sigma \bar{I}_{s c}(t)-\delta \bar{I}_{s c}(t) \\
& -\pi_{f} \bar{I}_{s c}(t) \text {, } \\
& \frac{d \bar{C}(t)}{d t}=\delta \bar{I}_{s c}(t)+\theta\left(1-u_{2}(t)\right) \bar{I}_{h}(t)-\gamma \bar{C}(t) \\
& -\pi_{f} \bar{C}(t), \\
& \frac{d \bar{R}_{f}(t)}{d t}=\sigma \bar{I}_{s c}(t)-\pi_{f} \bar{R}_{f}(t)-\alpha \bar{R}_{f}(t), \\
& \frac{d \bar{S}_{m}(t)}{d t}=\pi_{m}-c_{m} \beta_{f} \bar{I}_{h}(t) \bar{S}_{m}(t)-\pi_{m} \bar{S}_{m}(t) \\
& +\alpha \bar{R}_{m}(t) \text {, } \\
& \frac{d \bar{J}(t)}{d t}=c_{m} \beta_{f} \bar{I}_{h}(t) \bar{S}_{m}(t)-\zeta \bar{J}(t)-\pi_{m} \bar{J}(t), \\
& \frac{d \bar{R}_{m}(t)}{d t}=\zeta \bar{J}(t)-\pi_{m} \bar{R}_{m}(t)-\alpha \bar{R}_{m}(t) .
\end{aligned}
$$

\section{Objective Functional}

The aim of optimal control in this study was to minimize the number of populations infected with HPV and cervical cancer, as well as to minimize the control costs, namely

$Z\left[u_{1}, u_{2}\right]=\int_{0}^{T}\left[I_{h}+J+C+w_{1} u_{1}{ }^{2}(t)+w_{2} u_{2}{ }^{2}(t)\right] d t$.

$w_{1}$ is the weight of control costs in the form of vaccines with $u_{1}{ }^{2}(t)$ indicates the level of vaccination cost. $w_{2}$ is the weight of control costs in the form of screening with $u_{2}{ }^{2}(t)$ indicates the 
level of screening cost. Vaccination was given to susceptible female populations, while screening was carried out by populations of females infected with HPV with the appearance of several symptoms. Based on equation (4), we will look for $u_{1}^{*}, u_{2}^{*}$ which results in minimizing the objective functional value that is

$$
Z\left[u_{1}^{*}, u_{2}^{*}\right]=\min \left\{Z\left[u_{1}, u_{2}\right] \mid u_{1}, u_{2} \in \mathbb{V}\right\}
$$

with

$\mathbb{V}=\left\{\left(u_{1}(t), u_{2}(t)\right): 0 \leq u_{1}(t) \leq 1,0 \leq u_{2}(t) \leq 1\right\}$

\section{Hamiltonian Function}

Based on the objective functional (4) and the constraints on the equation (3) to get the optimal system, the Hamiltonian function is formed as follows.

$$
\begin{aligned}
& H=I_{h}+J+C+w_{1} u_{1}^{2}(t)+w_{2} u_{2}^{2}(t) \\
& +\lambda_{\bar{S}_{f}}\left(\pi_{f}-u_{1}(t) \bar{S}_{f}(t)-c_{f} \beta_{m} \bar{J}(t) \bar{S}_{f}(t)-\pi_{f} \bar{S}_{f}(t)\right. \\
& \left.+\alpha \bar{R}_{f}(t)\right) \\
& +\lambda_{\bar{V}}\left(u_{1}(t) \bar{S}_{f}(t)-(1-\varepsilon) c_{f} \beta_{m} \bar{J}(t) \bar{V}(t)-\pi_{f} \bar{V}\left(\bar{F}^{t}\right)\right) \\
& +\lambda_{\bar{I}_{h}}\left(c_{f} \beta_{m} \bar{J}(t) \bar{S}_{f}(t)+(1-\varepsilon) c_{f} \beta_{m} \bar{J}(t) \bar{V}(t)\right. \\
& \left.-u_{2}(t) \bar{I}_{h}(t)-\theta\left(1-u_{2}(t)\right) \bar{I}_{h}(t)-\pi_{f} \bar{I}_{h}(t)\right) \\
& +\lambda_{\bar{I}_{s c}}\left(u_{2}(t) \bar{I}_{h}-\sigma \bar{I}_{s c}(t)-\delta \bar{I}_{s c}(t)-\pi_{f} \bar{I}_{s c}(t)\right) \\
& +\lambda_{\bar{C}}\left(\delta \bar{I}_{s c}(t)+\theta\left(1-u_{2}(t)\right) \bar{I}_{h}(t)-\gamma \bar{C}(t)-\pi_{f} \bar{C}(t)\right) \\
& +\lambda_{\bar{R}_{f}}\left(\sigma \bar{I}_{s c}(t)-\pi_{f} \bar{R}_{f}(t)-\alpha \bar{R}_{f}(t)\right) \\
& +\lambda_{\bar{S}_{m}}\left(\pi_{m}-c_{m} \beta_{f} \bar{I}_{h}(t) \bar{S}_{m}(t)-\pi_{m} \bar{S}_{m}(t)+\alpha \bar{R}_{m}(t)\right) \\
& +\lambda_{\bar{J}}\left(c_{m} \beta_{f} \bar{I}_{h}(t) \bar{S}_{m}(t)-\zeta \bar{J}(t)-\pi_{m} \bar{J}(t)\right) \\
& +\lambda_{\bar{R}_{m}}\left(\zeta \bar{J}(t)-\pi_{m} \bar{R}_{m}(t)-\alpha \bar{R}_{m}(t)\right) \text {. }
\end{aligned}
$$

The Hamilton function reaches an optimal solution if the state equation, adjoint equation, and stationary conditions are fulfilled.

\section{State Equations}

The state equation for optimal control problems (4) with constraints (3) was obtained by differentiating the Hamiltonian function (5) with respect to each adjoint variable as follows.

$$
\begin{aligned}
& \frac{d \bar{S}_{f}}{d t}=\frac{\partial H}{d \lambda_{\bar{S}_{f}}}=\pi_{f}-u_{1}(t) \bar{S}_{f}(t)-c_{f} \beta_{m} \bar{J}(t) \bar{S}_{f}(t) \\
& -\pi_{f} \bar{S}_{f}(t)+\alpha \bar{R}_{f}(t), \\
& \frac{d \bar{V}}{d t}=\frac{\partial H}{d \lambda_{\bar{V}}}=u_{1}(t) \bar{S}_{f}(t)-(1-\varepsilon) c_{f} \beta_{m} \bar{J}(t) \bar{V}(t) \\
& -\pi_{f} \bar{V}(t) \\
& \frac{d \bar{I}_{h}}{d t}=\frac{\partial H}{d \lambda_{\bar{I}_{h}}}=c_{f} \beta_{m} \bar{J}(t) \bar{S}_{f}(t)+(1-\varepsilon) c_{f} \beta_{m} \bar{J}(t) \bar{V}(t) \\
& -u_{2}(t) \bar{I}_{h}(t)-\theta\left(1-u_{2}(t)\right) \bar{I}_{h}(t)-\pi_{f} \bar{I}_{h}(t),
\end{aligned}
$$

$$
\begin{aligned}
& \frac{d \bar{I}_{s c}}{d t}=\frac{\partial H}{d \lambda_{\bar{I}_{s c}}}=u_{2}(t) \bar{I}_{h}-\sigma \bar{I}_{s c}(t)-\delta \bar{I}_{s c}(t) \\
& -\pi_{f} \bar{I}_{s c}(t) \text {, } \\
& \frac{d \bar{C}}{d t}=\frac{\partial H}{d \lambda_{\bar{C}}}=\delta \bar{I}_{s c}(t)+\theta\left(1-u_{2}(t)\right) \bar{I}_{h}(t) \\
& -\gamma \bar{C}(t)-\pi_{f} \bar{C}(t), \\
& \frac{d \bar{R}_{f}}{d t}=\frac{\partial H}{d \lambda_{\bar{R}_{f}}}=\sigma \bar{I}_{s c}(t)-\pi_{f} \bar{R}_{f}(t)-\alpha \bar{R}_{f}(t), \\
& \frac{d \bar{S}_{m}}{d t}=\frac{\partial H}{d \lambda_{\bar{S}_{m}}}=\pi_{m}-c_{m} \beta_{f} \bar{I}_{h}(t) \bar{S}_{m}(t) \\
& -\pi_{m} \bar{S}_{m}(t)+\alpha \bar{R}_{m}(t), \\
& \frac{d \bar{J}}{d t}=\frac{\partial H}{d \lambda_{\bar{J}}}=c_{m} \beta_{f} \bar{I}_{h}(t) \bar{S}_{m}(t)-\zeta \bar{J}(t)-\pi_{m} \bar{J}(t), \\
& \frac{d \bar{R}_{m}}{d t}=\frac{\partial H}{d \lambda_{\bar{R}_{m}}}=\zeta \bar{J}(t)-\pi_{m} \bar{R}_{m}(t)-\alpha \bar{R}_{m}(t) \text {, }
\end{aligned}
$$

\section{Adjoint Equations}

The adjoint equation for the optimal control problem (4) with the constraint (3) was obtained by differentiating the Hamiltonian function (5) with respect to each state variable as follows.

$$
\begin{gathered}
\frac{d \lambda_{\bar{S}_{f}}}{d t}=-\frac{\partial H}{\partial \bar{S}_{f}}=\left(u_{1}(t)+c_{f} \beta_{m} \bar{J}(t)+\pi_{f}\right) \lambda_{\bar{s}_{f}} \\
\quad-u_{1}(t) \lambda_{\bar{V}}-c_{f} \beta_{m} \bar{J}(t) \lambda_{\bar{I}_{h^{\prime}}} \\
\frac{d \lambda_{\bar{V}}}{d t}=-\frac{\partial H}{\partial \bar{V}}=\begin{array}{c}
\left(\pi_{f}+(1-\varepsilon) c_{f} \beta_{m} \bar{J}(t)\right) \lambda_{\bar{V}} \\
-(1-\varepsilon) c_{f} \beta_{m} \bar{J}(t) \lambda_{\bar{I}_{h}},
\end{array}
\end{gathered}
$$

$\frac{d \lambda_{\bar{I}_{h}}}{d t}=-\frac{\partial H}{\partial \bar{I}_{h}}=-1+\left(u_{2}(t)+\theta\left(1-u_{2}(t)\right)+\pi_{f}\right) \lambda_{\bar{I}_{h}}$

$$
-u_{2}(t) \lambda_{\bar{I}_{s c}}-\theta\left(1-u_{2}(t)\right) \lambda_{\bar{C}}
$$$$
+c_{m} \beta_{f} \bar{S}_{m}(t) \lambda_{\bar{S}_{m}}-c_{m} \beta_{f} \bar{S}_{m}(t) \lambda_{\bar{J}}
$$

$\frac{d \lambda_{\bar{I}_{s c}}}{d t}=-\frac{\partial H}{\partial \bar{I}_{s c}}=\left(\sigma+\delta+\pi_{f}\right) \lambda_{\bar{I}_{s c}}-\delta \lambda_{\bar{C}}-\sigma \lambda_{\bar{R}_{f}}$,

$\frac{d \lambda_{\bar{C}}}{d t}=-\frac{\partial H}{\partial \bar{C}}=-1+\left(\pi_{f}+\gamma\right) \lambda_{\bar{C}}$,

$\frac{d \lambda_{\bar{R}_{f}}}{d t}=-\frac{\partial H}{\partial \bar{R}_{f}}=\pi_{f} \lambda_{\bar{R}_{f}}-\alpha \lambda_{\bar{S}_{f}}+\alpha \lambda_{\bar{R}_{f}}$,

$\frac{d \lambda_{\bar{S}_{m}}}{d t}=-\frac{\partial H}{\partial \bar{S}_{m}}=\left(c_{m} \beta_{f} \bar{I}_{h}(t)+\pi_{m}\right) \lambda_{\bar{S}_{m}}-c_{m} \beta_{f} \bar{I}_{h}(t) \lambda_{\bar{J}}$,

$\frac{d \lambda_{\bar{J}}}{d t}=-\frac{\partial H}{\partial \bar{J}}=-1+c_{f} \beta_{m} \bar{S}_{f}(t) \lambda_{\bar{S}_{f}}$

$$
\begin{aligned}
& +(1-\varepsilon) c_{f} \beta_{m} \bar{V}(t) \lambda_{\bar{V}} \\
& -\left(c_{f} \beta_{m} \bar{S}_{f}(t)+(1-\varepsilon) c_{f} \beta_{m} \bar{V}(t)\right) \lambda_{\bar{I}_{h}}
\end{aligned}
$$$$
+\left(\zeta+\pi_{m}\right) \lambda_{\bar{J}}-\zeta \lambda_{\bar{R}_{m}}
$$

$\frac{d \lambda_{\bar{R}_{m}}}{d t}=-\frac{\partial H}{\partial \bar{R}_{m}}=\pi_{m} \lambda_{\bar{R}_{m}}-\alpha \lambda_{\bar{S}_{m}}+\alpha \lambda_{\bar{R}_{m}}$, 
with transversality conditions $\lambda_{\bar{S}_{f}}(T)=\lambda_{\bar{V}}(T)=$ $\lambda_{\bar{I}_{h}}(T)=\lambda_{\bar{I}_{s c}}(T)=\lambda_{\bar{C}}(T)=\lambda_{\bar{R}_{f}}(T)=\lambda_{\bar{S}_{m}}(T)=$ $\lambda_{\bar{J}}(T)=\lambda_{\bar{R}_{m}}(T)=0$.

\section{Stationer Conditions}

Stationer conditions for optimal control problems (4) with constraints (3) was obtained by differentiating the Hamiltonian function (5) toward variables $u_{1}, u_{2}$ as follows.

$$
\begin{aligned}
\frac{\partial H}{\partial u_{1}}= & 2 w_{1} u_{1}(t)-\bar{S}_{f}(t) \lambda_{\bar{S}_{f}}+\bar{S}_{f}(t) \lambda_{\bar{V}}=0, \\
u_{1}^{*}(t)= & \frac{\left(\lambda_{\bar{S}_{f}}-\lambda_{\bar{V}}\right) \bar{S}_{f}^{*}(t)}{2 w_{1}}, \\
\frac{\partial H}{\partial u_{2}}= & 2 w_{2} u_{2}(t)-\bar{I}_{h}(t) \lambda_{\bar{I}_{h}}+\theta \bar{I}_{h}(t) \lambda_{\bar{I}_{h}} \\
& +\bar{I}_{h}(t) \lambda_{\bar{I}_{s c}}-\theta \bar{I}_{h}(t) \lambda_{\bar{C}}=0, \\
u_{2}^{*}(t)= & \frac{\left(\lambda_{\bar{I}_{h}}-\lambda_{\bar{I}_{s c}} \bar{I}_{h}^{*}(t)+\left(\lambda_{\bar{C}}-\lambda_{\bar{I}_{h}}\right) \theta \bar{I}_{h}^{*}(t)\right.}{2 w_{2}} .
\end{aligned}
$$

Given that $0 \leq u_{1}(t) \leq 1$ and $0 \leq u_{2}(t) \leq 1$, thus we get the equation as follows.

$$
\begin{array}{r}
u_{1}^{*}(t)=\left\{\begin{array}{cr}
0, & u_{1}(t) \leq 0 \\
u_{1}(t), & 0<u_{1}(t)<1 \\
1, & u_{1}(t) \geq 1 .
\end{array}\right. \\
u_{2}^{*}(t)=\left\{\begin{array}{cr}
0, & u_{2}(t) \leq 0 \\
u_{2}(t), & 0<u_{2}(t)<1 \\
1, & u_{2}(t) \geq 1 .
\end{array}\right.
\end{array}
$$

In compact notation,

$$
\begin{aligned}
& u_{1}^{*}(t)=\min \left\{\max \left[0, \frac{\left(\lambda_{\bar{S}_{f}}-\lambda_{\bar{V}}\right) \bar{S}_{f}^{*}(t)}{2 w_{1}}\right], 1\right\}, \\
& u_{2}^{*}(t)=\min \left\{\max \left[0, \frac{\left(\lambda_{\bar{I}_{h}}-\lambda_{\bar{I}_{s c}}\right) \bar{I}_{h}^{*}(t)+\left(\lambda_{\bar{C}}-\lambda_{\bar{I}_{h}}\right) \theta \bar{I}_{h}^{*}(t)}{2 w_{2}}\right], 1\right\}
\end{aligned}
$$

\section{Optimal Solution}

The optimal solution was obtained by substituting the optimal control $\vec{u}^{*}$ to the system of state equations (6) and adjoint equations (7) so that the equation system was obtained as follows.

$$
\begin{gathered}
\frac{d \bar{S}_{f}^{*}(t)}{d t}=\pi_{f}-u_{1}^{*}(t) \bar{S}_{f}^{*}(t)-c_{f} \beta_{m} \bar{J}^{*}(t) \bar{S}_{f}^{*}(t) \\
-\pi_{f} \bar{S}_{f}^{*}(t)+\alpha \bar{R}_{f}^{*}(t), \\
\frac{d \bar{V}^{*}(t)}{d t}=u_{1}^{*}(t) \bar{S}_{f}^{*}(t)-(1-\varepsilon) c_{f} \beta_{m} \bar{J}^{*}(t) \bar{V}^{*}(t) \\
\quad-\pi_{f} \bar{V}^{*}(t), \\
\frac{d \bar{I}_{h}^{*}(t)}{d t}=\begin{array}{c}
c_{f} \beta_{m} \bar{J}^{*}(t) \bar{S}_{f}^{*}(t)+(1-\varepsilon) c_{f} \beta_{m} \bar{J}^{*}(t) \bar{V}^{*}(t) \\
-u_{2}^{*}(t) \bar{I}_{h}^{*}(t)-\theta\left(1-u_{2}^{*}(t)\right) \bar{I}_{h}^{*}(t) \\
-\pi_{f} \bar{I}_{h}^{*}(t),
\end{array}
\end{gathered}
$$

$$
\begin{aligned}
& \frac{d \bar{I}_{s c}^{*}(t)}{d t}=u_{2}^{*}(t) \bar{I}_{h}^{*}-\sigma \bar{I}_{s c}^{*}(t)-\delta \bar{I}_{s c}^{*}(t)-\pi_{f} \bar{I}_{s c}^{*}(t), \\
& \frac{d \bar{C}^{*}(t)}{d t}=\delta \bar{I}_{s c}^{*}(t)+\theta\left(1-u_{2}^{*}(t)\right) \bar{I}_{h}^{*}(t) \\
& -\gamma \bar{C}^{*}(t)-\pi_{f} \bar{C}^{*}(t) \text {, } \\
& \frac{d \bar{R}_{f}^{*}(t)}{d t}=\sigma \bar{I}_{s c}^{*}(t)-\pi_{f} \bar{R}_{f}^{*}(t)-\alpha \bar{R}_{f}^{*}(t), \\
& \frac{d \bar{S}_{m}^{*}(t)}{d t}=\pi_{m}-c_{m} \beta_{f} \bar{I}_{h}^{*}(t) \bar{S}_{m}^{*}(t)-\pi_{m} \bar{S}_{m}^{*}(t) \\
& +\alpha \bar{R}_{m}^{*}(t) \text {, } \\
& \frac{d \bar{J}^{*}(t)}{d t}=c_{m} \beta_{f} \bar{I}_{h}^{*}(t) \bar{S}_{m}^{*}(t)-\zeta \bar{J}^{*}(t)-\pi_{m} \bar{J}^{*}(t), \\
& \frac{d \bar{R}_{m}^{*}(t)}{d t}=\zeta \bar{J}^{*}(t)-\pi_{m} \bar{R}_{m}^{*}(t)-\alpha \bar{R}_{m}^{*}(t), \\
& \frac{d \lambda_{\bar{s}_{f}}}{d t}=\left(u_{1}^{*}(t)+c_{f} \beta_{m} \bar{J}^{*}(t)+\pi_{f}\right) \lambda_{\bar{s}_{f}} \\
& -u_{1}^{*}(t) \lambda_{\bar{V}}-c_{f} \beta_{m} \bar{J}^{*}(t) \lambda_{\bar{I}_{h}}, \\
& \frac{d \lambda_{\bar{V}}}{d t}=\left(\pi_{f}+(1-\varepsilon) c_{f} \beta_{m} \bar{J}^{*}(t)\right) \lambda_{\bar{V}} \\
& -(1-\varepsilon) c_{f} \beta_{m} \bar{J}^{*}(t) \lambda_{\bar{I}_{h}} \text {, } \\
& \frac{d \lambda_{\bar{I}_{h}}}{d t}=-1+\left(u_{2}^{*}(t)+\theta\left(1-u_{2}^{*}(t)\right)+\pi_{f}\right) \lambda_{\bar{I}_{h}} \\
& -u_{2}^{*}(t) \lambda_{\bar{I}_{s c}}-\theta\left(1-u_{2}^{*}(t)\right) \lambda_{\bar{C}} \\
& +c_{m} \beta_{f} \bar{S}_{m}^{*}(t) \lambda_{\bar{S}_{m}}-c_{m} \beta_{f} \bar{S}_{m}^{*}(t) \lambda_{\bar{J}} \text {, } \\
& \frac{d \lambda_{\bar{I}_{s c}}}{d t}=\left(\sigma+\delta+\pi_{f}\right) \lambda_{\bar{I}_{s c}}-\delta \lambda_{\bar{C}}-\sigma \lambda_{\bar{R}_{f}} \\
& \frac{d \lambda_{\bar{C}}}{d t}=-1+\left(\pi_{f}+\gamma\right) \lambda_{\bar{C}} \text {, } \\
& \frac{d \lambda_{\bar{R}_{f}}}{d t}=\pi_{f} \lambda_{\bar{R}_{f}}-\alpha \lambda_{\bar{s}_{f}}+\alpha \lambda_{\bar{R}_{f}} \\
& \frac{d \lambda_{\bar{S}_{m}}}{d t}=\left(c_{m} \beta_{f} \bar{I}_{h}^{*}(t)+\pi_{m}\right) \lambda_{\bar{S}_{m}}-c_{m} \beta_{f} \bar{I}_{h}^{*}(t) \lambda_{\bar{J}}, \\
& \frac{d \lambda_{\bar{I}}}{d t}=-1+c_{f} \beta_{m} \bar{S}_{f}^{*}(t) \lambda_{\bar{S}_{f}}+(1-\varepsilon) c_{f} \beta_{m} \bar{V}^{*}(t) \lambda_{\bar{V}} \\
& -\left(c_{f} \beta_{m} \bar{S}_{f}^{*}(t)\right. \\
& \left.+(1-\varepsilon) c_{f} \beta_{m} \bar{V}^{*}(t)\right) \lambda_{\bar{I}_{h}} \\
& +\left(\zeta+\pi_{m}\right) \lambda_{\bar{J}}-\zeta \lambda_{\bar{R}_{m}}, \\
& \frac{d \lambda_{\bar{R}_{m}}}{d t}=\pi_{m} \lambda_{\bar{R}_{m}}-\alpha \lambda_{\bar{S}_{m}}+\alpha \lambda_{\bar{R}_{m}} \text {, }
\end{aligned}
$$

with boundary conditions $\bar{S}_{f}(0)=\bar{S}_{f_{0}}, \bar{V}(0)=$ $\bar{V}_{0}$,

$\bar{I}_{h}(0)=\bar{I}_{h_{0}}, \bar{I}_{s c}(0)=\bar{I}_{s c_{0}}, \bar{C}(0)=\bar{C}_{0}, \bar{R}_{f}(0)=\bar{R}_{f_{0}}$, $\bar{S}_{m}(0)=\bar{S}_{m_{0}}, \bar{J}(0)=\bar{J}_{0}, \bar{R}_{m}(0)=\bar{R}_{m_{0}}, \lambda_{\bar{S}_{f}}(T)=$ $\lambda_{\bar{V}}(T)=\lambda_{\bar{I}_{h}}(T)=\lambda_{\bar{I}_{S C}}(T)=\lambda_{\bar{C}}(T)=\lambda_{\bar{R}_{f}}(T)=$ $\lambda_{\bar{S}_{m}}(T)=\lambda_{\bar{J}}(T)=\lambda_{\bar{R}_{m}}(T)=0$.

\section{Numerical method and simulations}

Numerical simulations are conducted by using initial value $\overline{\mathrm{S}}_{\mathrm{f}}(0)=0.3, \overline{\mathrm{V}}(0)=0.2, \overline{\mathrm{I}}_{\mathrm{h}}(0)=$ $0.1, \bar{I}_{s c}(0)=0.1, \bar{C}(0)=0.2, \bar{R}_{f}(0)=$ $0.1, \bar{S}_{m}(0)=\bar{S}_{m}(0)=0.5, \bar{J}(0)=0.3, \bar{R}_{m}(0)=$ 0.2 . 
The simulation result with and without control is presented in Figure 2. The graph solution is shown with the weight values of controls $w_{1}=$ $0.5, \quad w_{2}=0.2$, and parameter values, as presented in Table 1. Based on Figure 2, giving a combination of vaccination and screening control have a positive effect on the spread of HPV infection. It appears that the combination of controls can reduce the spread of HPV infection. It can be seen by decreasing the number of subpopulations of females infected with HPV. Before being given control, the number of females infected with HVP is increased. However, after being given control, the number of infected females decreases very significantly. It also results in a decreasing number of cervical cancers. Meanwhile, the combination of controls also has an impact on male subpopulation. These controls are also able to reduce the infected male subpopulation.
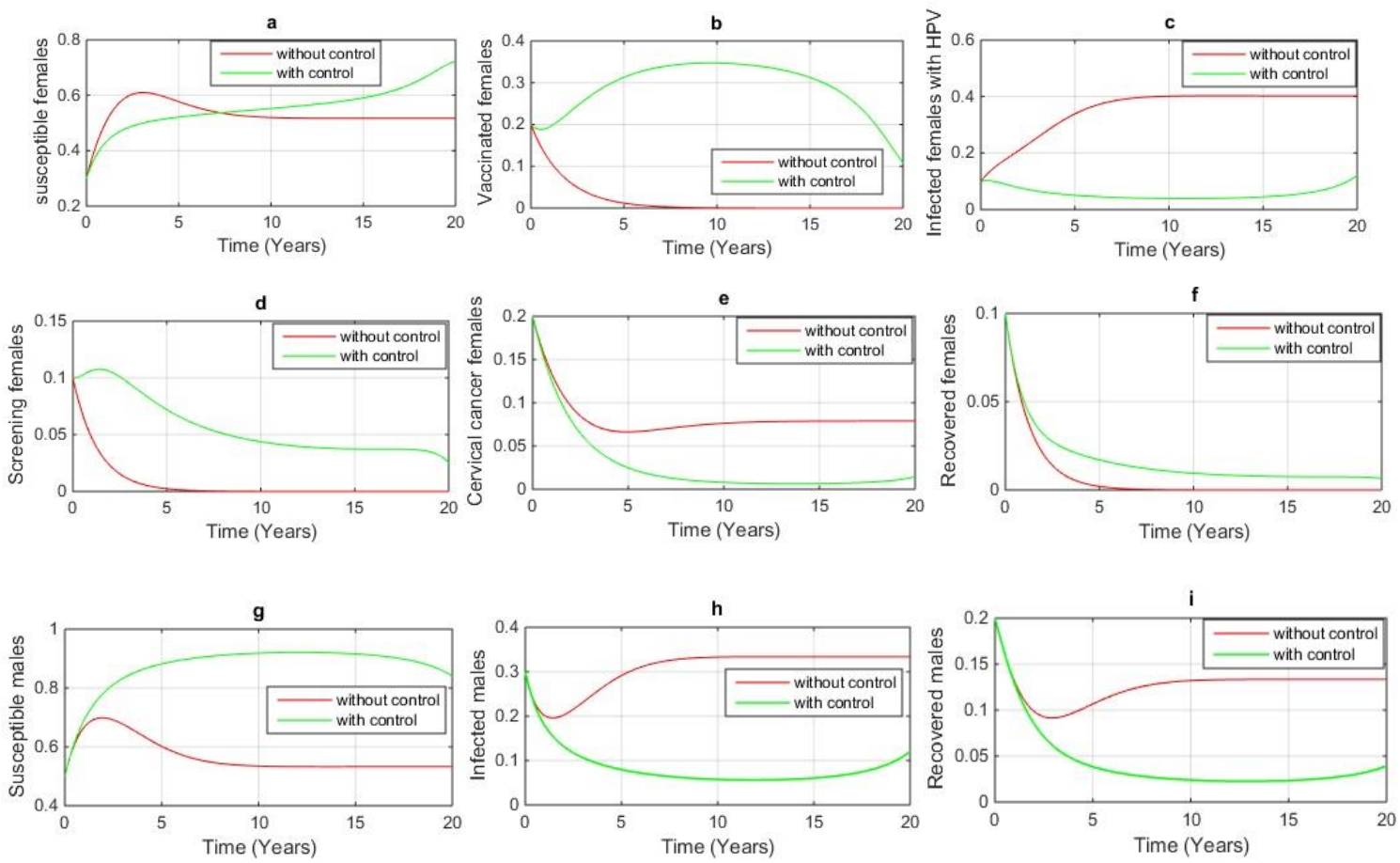

Figure 2. The behavior of cervical cancer model for $t \in[0,20]$

Table 1. Parameter values

\begin{tabular}{|c|c|c|c|}
\hline Parameter & Description & Value & References \\
\hline$\pi_{f}\left(\pi_{m}\right)$ & $\begin{array}{l}\text { Number of recruitment rate of a sexually- } \\
\text { active subpopulation of female (male) }\end{array}$ & 0.5 & assumed \\
\hline$\mu_{f}\left(\mu_{m}\right)$ & $\begin{array}{l}\text { The death rate of the subpopulation of } \\
\text { female (male) }\end{array}$ & 0.1 & assumed \\
\hline$\beta_{f}\left(\beta_{m}\right)$ & $\begin{array}{l}\text { Probability of transfer infection from } \\
\text { females to males (males to females) }\end{array}$ & 0.7 & {$[6]$} \\
\hline$c_{f}\left(c_{m}\right)$ & $\begin{array}{l}\text { The average number of sexual contacts of } \\
\text { female for males (male for females) }\end{array}$ & 2 & {$[6]$} \\
\hline$\phi$ & Vaccination rate & 0 & assumed \\
\hline$\varepsilon$ & Efficacy vaccines & 0.8 & [6] \\
\hline$p$ & Screening rate & 0 & assumed \\
\hline$\theta$ & $\begin{array}{l}\text { Probability of infected males with HPV can } \\
\text { be infected with cervical cancer }\end{array}$ & 0.1 & assumed \\
\hline$\sigma$ & The recovery rate of females infected & 0.2 & assumed \\
\hline$\zeta$ & The recovery rate of males infected & 0.4 & assumed \\
\hline$\delta$ & $\begin{array}{l}\text { Probability of screening females can be } \\
\text { infected with cervical cancer }\end{array}$ & 0.05 & assumed \\
\hline$\gamma$ & $\begin{array}{l}\text { Cervical cancer-induced mortality rate in } \\
\text { females }\end{array}$ & 0.01 & assumed \\
\hline$\alpha$ & The rate of immune loss & 0.5 & assumed \\
\hline
\end{tabular}


Furthermore, Figure 3 shows the control profile in the form of vaccination and screening. Both of them were used in reducing the number of infected female subpopulations and the infected male subpopulations, as well as the number of cervical cancers. It appears that the vaccination control given is lower than the screening control. It can be influenced by the ability of vaccination given, which has enough impact to reduce the number of infected subpopulations. Meanwhile, screening control must be given in a higher dose to effectively help in suppressing the occurrence of infection.

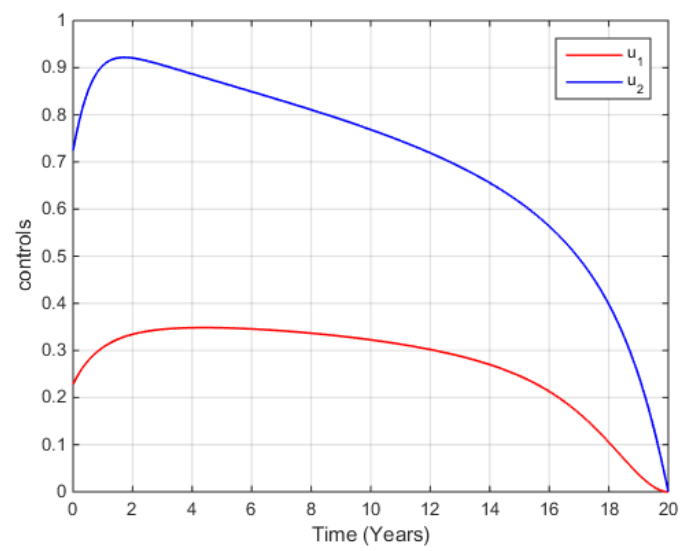

Figure 3. The control profile of cervical cancer model

\section{CONCLUSION}

In this study, optimal control of the cervical cancer model has been carried out. Two controls were used in this model, namely vaccination and screening. Based on the results of the study, the provision of these two controls has a positive impact as it can reduce the number of infected subpopulations, both in the subpopulation of infected females and in the subpopulation of infected males. In addition, the administration of this control also leads to a reduced rate of cervical cancer in the female population.

\section{REFERENCES}

[1]. Diananda, R. 2008. Mengenal seluk beluk kanker. Katahati. Yogyakarta.

[2]. Centers for Disease Control and Prevention (CDC). 2015. Prevention Human Papillomavirus (HPV). Available at: https://www.cdc.gov/std/HPV/STDFactHPV.htm.
[3]. Rasjidi, I. 2009. Epidemiologi kanker serviks. Indonesian Journal of Cancer. 3. 103-108.

[4]. Centers for Disease Control and Prevention (CDC). 2019. Cervical cancer awareness month social media toolkit. Cancer Center. The George Washington University.

[5]. Pongsumpun, P. 2014. Mathematical model of cervical cancer due to human papillomavirus infection. Available at: http://www.inase.org/library/2014/athens /bypaper/MMCTSE/MMCTSE-28.pdf

[6]. Malik, T.M., Imran., R. Jayaraman. 2016. Optimal control with multiple human papillomavirus vaccines. J. Theor. Biol. 393. 179-193.

[7]. Sado, E.A. 2019. Mathematical modeling of cervical cancer with HPV transmission and vaccination. Sci. J. Appl. Math. Stat. 7. 21-25.

[8]. Saldana, F., A. Korobeinikov, I. Barradas, 2019. Optimal control against the human papillomavirus protection versus eradication of the infection. Abstr. Appl. Anal. 10. 1-13.

[9]. Lenhart, S., J.T. Workman. 2007. Optimal control applied to biological models. CRC Press. London.

[10]. Bzhalava, D., P. Guan, S. Franceschi, J. Dillner, G. Clifford. 2013. A systematic review of the prevalence of mucosal and cutaneous human papillomavirus types. J. Virol. 445. 224-23. 